\title{
Discurso de viajes y sentido de la alteridad*
}

Wladimir Krysinski

Es posible que la relación con los demás, hoy, que puede ser una relación psicológica, social, etc. tenga un poco de esa dimensión transitiva, transversal, vectorial; a rigor, que más no haga que circundar el deseo de los demás, en relación con los demás. Es posible una relación fuerte con los demás, con un deseo propio, un descubrimiento del otro, del afecto, de todo lo que podría constituir pasiones, con una cierta intensidad. Y también se puede imaginar el otro como lugar de la desposesión, pura y simple. Esto

significa que el otro existe, pero es destinado para ser cruzado, en cierto modo, se puede vivir en el deseo del otro, sino también en el exilio en otra dimensión, de fondo holográfico. Es casi un holograma en ese momento, que usted puede pasar.

Jean Baudrillard (1994: 91-92)

* Esta versión en español corresponde al texto presentado en francés en el Coloquio «Christian Metz et la théorie du cinéma». Centre Culturel International de Cerisy-la-Salle, Francia, 29 de junio de 1989. Versiones anteriores de este texto fueron presentadas en ambas lenguas. La traducción pertenece a Vaniery P. Amorim (N. del E.). 


\section{Entre el discurso y la narración: el viaje como un operador cognitivo}

\section{1}

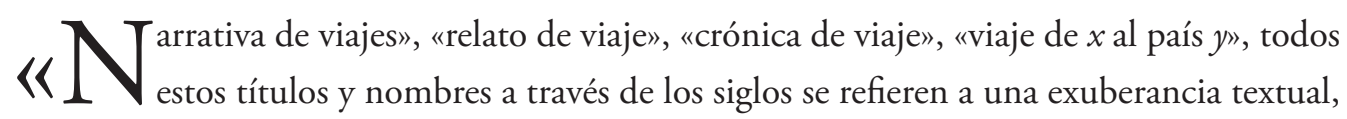
según el hecho de que el movimiento formó el mundo y la humanidad. El viaje también modeló la cultura, y, en algunos casos, como Portugal, fue la propia sociedad que la formó. El viaje es consubstancial a la historia, la mitología y la literatura. Siempre renovable, frente a un lugar variable por excelencia, el viaje ofrece la literatura una de sus principales materias primas.

De Homero a Elias Canetti, ${ }^{1}$ a través de Mendes Pinto, Swift, Sterne, Diderot, Chateaubriand, Almeida Garrett, Melville, Joyce, Michel Butor, Henri Michaux, Darcy Ribeiro $^{2}$ y Allen Ginsberg, ${ }^{3}$ el viaje mantiene el progreso del discurso literario. El viaje determina los informes y las formas simbólicas que están entre el viajante-narrador, el espacio y el tiempo. Estos relatos y formas son sostenidos por un discurso que introduce su subjetividad en la objetividad de la realidad, de lo histórico, social y político.

En el ámbito heterogéneo de los hechos literarios, sería tentador proponer una tipología de las narraciones de viaje, ¿pasar en el bisturí el genológico o genealógico? Esta vasta literatura que cubre una amplia variedad de formas y estructuras. Mi objetivo será diferente. Me gustaría mostrar cómo el viaje — como tema invariable — está pasando por una serie de avatares discursivos, cómo se complejifica como discurso en la perspectiva de

\footnotetext{
1 Véase Die Stimmen von Marrakesch. Nos referimos aquí a la edición en portugués, especialmente para el capítulo "Visita a Mellah», en la que el narrador relata: "[Yo] tenía la impresión de que no había realmente logrado el objetivo de mi viaje. No más quería salir ya había estado aquí cientos de años, pero se olvidaría. Ahora reencontraría la plaza. En este sentido, ofrecían a la densidad y el calor de la vida que ha sentido siempre dentro de mí. Yo era la plaza donde yo estaba. Creo que todavía yo he sido» (Canetti 1987: 55).

2 En Darcy Ribeiro (1976), en la cuarta de cubierta de esta versión, se indica que se trata de una novela en la que el autor retoma el tema del viaje iniciático en las aviezas. Darcy Ribeiro «pone a entender la manera en que directamente la voz de los indios de la Amazonía, el grito de una civilización que está agonizante porque no está en condiciones de adaptarse a las normas tecnológicas de nuestra sociedad. [...] Isaías parte en el seminario en Roma. Obsesionado por la memoria de su pueblo, roído de duda, pide para ver su familia antes de ser ordenado sacerdote. No más volverá a la vida con los blancos y convertíase de nuevo Ava, con sujeción a los requisitos atavistas y se tornará jefe de su tribu».

${ }_{3}^{3}$ Ginsberg en Journaux indiens representa un viaje a la vez real y onírico, y es un notable pot-pourri (véase La opinión de Yves Le Pellee, que lo dijo en la portada cuatro).
} 
una confrontación de las múltiples alteridades y, además, exponer cuántos en el espacio de la modernidad se convierten en operadores cognitivos que producen sin cesar un efecto estimulante en sujetos de enunciación en la búsqueda de saber. Lo que me interesa especialmente es cómo los discursos de los viajes generan la estructura tripartita de la literatura definida por Roland Barthes como mathesis, mimesis y semiosis, es decir, como saber, representación y significado (Barthes 1978: 17).

Las discursividades y la narrativa que se organizan en torno del viaje parecen poner en práctica las intuiciones y los locales de las críticas de Roland Barthes sobre estos tres elementos, los que constituirían la literatura. A través de las narrativas de viajes, se pueden observar, de hecho, que la literatura moviliza un saber que «no es nunca ni entero ni último»; la literatura según Barthes «no dice que sabe algo, pero sabe de algo» (Barthes 1978:18-19).

\section{2}

$\mathrm{E}^{1}$ infinita: a través de la escritura, el conocimiento refleja incesantemente a respecto del saber, de acuerdo con el discurso que ya no es epistemológico, más dramático» (Barthes 1978: 19). Sí, pues el viaje es un operador cognitivo, lo es mientras la dialéctica es el símbolo de intercambio semántico entre la topología de desplazamiento y la multiplicación de los signos que el viajante-narrador produce para superar las alteridades con que se enfrenta. El viaje opera recursivamente de algún modo como un operador de la cognición, y eso en la medida en que el narrador, que está constantemente en una posición exotópica, ${ }^{4}$ es decir, fuera con relación al objeto de su mirada, para expresar su curiosidad y su deseo de empatía y, al mismo tiempo, desenrollar su acto representativo, mimético, para situar el sentido. Esto no puede materializarse a menos que en la forma de un intercambio de una reciprocidad de signos entre lo extraño y lo que es familiar. ${ }^{5}$ Este intercambio lleva a menudo a poner el otro en una ficción a un precio de los estereotipos que contienen la sensación en los esbozos narrativos y discursivos recibidos por la comunidad de lectores a los que pertenece el viajante-narrador.

\footnotetext{
${ }^{4}$ Véase Bajtín (1997: 6). En el prólogo, Tzvetan Todorov aborda principalmente el «tema de la relación entre el creador y la creación de seres en este, o, como dijo Bajtín, entre el autor y el héroe». Todorov caracteriza así: «En términos generales, es decir, que una vida es un sentimiento, y con un ingrediente que hace posible la construcción de la estética, sólo es vista desde el exterior, en su conjunto. Deben estar plenamente incluidos en horizonte de alguna persona, y para el personaje, ese alguien más es, por supuesto, el autor: es lo que Bakhtin llama a la "exotopia" de este último".

5 Véase en especial Le Clézio 1975.
} 
Tantos viajes narrativizados, tematizados y problematizados demuestran que el conocimiento está limitado por las limitaciones subjetivas y espacio-temporales de una comunicación específica. Esto contrasta con el narrador-observador-analista y el espacio experimentado por la mirada, así como los desafíos de las alteridadades determinan el umbral y el punto de llegada del conocimiento. Esta situación de comunicación produce el efecto del sentido que el viajante-narrador debe hacer frente a la tensión entre la experiencia metaorgánica del espacio extranjero y de las características intrínsecas de ese espacio, que no se puede penetrar sólo aproximadamente.

\section{3}

$\mathrm{E}^{\mathrm{s}}$

s especialmente importante ver que en la modernidad, que lleva de Cervantes a Canetti, se convierta noológicamente por la internalización y por mitopoese en los ojos del narrador poeta o el poeta-narrador, sino también en la conciencia reflexiva de la etnografía. Los universos étnicos y psicológicos mantienen siempre una cuota del hermetismo: jamás será posible conocerlos por completo. La alteridad es fundamentalmente irreconocible. El viaje aparece como la revelación tangible de exotopias que afrenta la reciprocidad de la mirada que se ve y se está viendo. Por lo tanto, el viaje sólo puede dramatizar y problematizar esta no permeabilidad de los mundos representados. El otro es tan otro que el yo jamás podría conocerlo.

En el umbral de nuestra modernidad, este caso límite de la alteridad es investigado narrativamente por Joseph Conrad en Heart of Darkness (El corazón de las tinieblas). Este texto, capital por la temática particular de un viaje particular a los confines del África negro, levanta las cuestiones más importantes del siglo xx sobre la identidad y la alteridad, en vista de la oposición entre el mundo imperialista de los occidentales y el mundo colonizado de los salvajes, relegados a la condición de alteridad desdeńable, irreconocible y desconocida, puesta entre paréntesis, si no rechazada por el mundo occidental. Los ecos de Conrad de la narración maiéutica son audibles no sólo en el Diario de Bronislaw Malinowski, sino también en L'Afrique fantasma (África fantasma), de Michel Leiris, y en Tristes Tropiques (Tristes trópicos), de Claude Lévi-Strauss. La obra poética y los viajes paradójicos de Henri Michaux son un contrapunto absolutamente fascinante a estos viajes-interrogaciones, de estas problematizaciones de la alteridad. 
El exotopia, que es el lugar del mirar sobre la alteridad, es una posición cognitiva que permite conocer al otro, al menos exteriormente, a través de los invariantes de la humanidad, tales como la religión, la lengua, el amor, el comportamiento de cuerpo y la proxémica. Esta dialéctica relativiza lo familiar, mientras cuestiona el extranjero. El viaje centrado en un objetivo es superior a lo cotidiano. Ella conduce al otro lado. Vería en Calvino o Le Clézio este esfuerzo ejemplar que pretende el conocimiento del otro a través de la sorpresa de su espacio.

Tenemos que ver, sin embargo, que en el espacio discursivo de los viajes, nuestra modernidad todavía alberga discursos, tales como el de Michel Butor, de Jean Baudrillard y Walter Abish.

i Baudrillard en Amérique (América) extiende la tradición de los viajes filosóficos, analíticos
y críticos; Butor, en Mobile (Móvil), y Abisa, en Alphabetical Africa (África alfabética), domestican la complejidad e «intraducibilidad» del espacio extranjero por un abordaje combinatorio. La alteridad es vista como una entidad integrada. Es «modalizada» por un saber ocular, es también antropológica y mítica, a veces estadística, comprende incluso en grandes textos, sobre todo en Butor, Calvino y Abish, que llevan la cuestión de la identidad y la alteridad a otro nivel del espacio cosmológico, que confraterniza con todos, sin distinción. Abish alfabéticamente investiga el espacio africano, a punto de hacer de ella una combinatoria disciplina catalogada; mientras que Butor representa los Estados Unidos a través de un gran recorte, construcción a la similitud de los móviles de Calder, lo que representa la omnipresencia de lo visible y lo tecnológico.

Así viendo, el sentido de la alteridad está proveído con nuevos signos. Parece que en el siglo xx, el viaje desempeñó hasta el final su papel del operador cognitivo. El mosaico semántico de los signos distribuyó miradas e intencionalidades delante de la situación comunicación arquetípica del narrador-viajante, como la definió el yo narrante, el espacio recorrido e investigado, el otro puesto como la ignorancia fundamental o como un teatro que describe la diferencia. Sería ilusorio pensar que toda la literatura de viajes de la modernidad se basa en esos supuestos. Sin embargo, las posiciones respectivas de Conrad, Malinowski, Leiris (L'Afrique fantasma), Michaux (Voyage en Grande Garabagne [Viajes por el Gran Garabagne]), en Ailleurs (En otro sitio), de Lévi-Strauss (Tristes Tropiques), de Darcy Ribeiro (Maira) y Le Clézio (Voyages de l'Autre Côté [Viajes en el otro lado]) crean, al que parece ser, un paradigma 
en el que la alteridad es una problematización máxima que pasa por la experiencia ocular del otro y que destaca la distancia etnográfica y la no comunicabilidad.

La mirada del escritor y del etnógrafo se percibe en la encrucijada de un saber siempre parcial, de un espacio intransitable cuando el proyecto del Occidente moderno se deshace, el de la emancipación de la humanidad, claro en el siglo de las luces. Es preciso entonces repensar las incertidumbres de algunos discursos de viaje, así como determinados sentidos de la alteridad. Este es el precio que sí accede a una mejor y más completa comprensión del siglo. Este entendimiento es a través de la valoración de la tensión fundadora de la moderna literatura entre la disposición de conocer y la complejidad del saber.

\section{Paradigmas paralelos y concurrentes:} subjetividad y alteridades etnográficas

\section{1}

$\mathrm{E}_{\mathrm{c}}^{\mathrm{n}}$ nu análisis de Les Indes noires (Las Indias negras), de Julio Verne, Michel Serres define l viaje de la siguiente manera:

Cada viaje está indexado con una leyenda de tres entradas. Es un desplazamiento en el espacio, guiado, como hemos visto, en el plano vertical u horizontal del avión: ruta de apropiación de la tierra, visita, exploración, giro. Se trata de una investigación científica, que lentamente se extiende por el currículum enciclopédico: es la razón por la que el viajante es (o va acompańado o precedido por) un ingeniero o un científico, geólogo, etc. El objetivo es encontrar el lugar donde un problema se resuelva por sí mismo, encontrar el lugar donde el conocimiento está presente. (Serres 1986: 292)

Esta definición se aplica a los discursos de viajes aquí propuestos, conforme se encuentra esa misma dinámica cognitiva en ellos, que marca las relaciones entre el desplazamiento y lo que Michel Serres llama cierto conocimiento presente. Será visto como diferentes paradigmas de los discursos de viajes que estructuran a la narrativa, al espacio y al conocimiento. Desde el comienzo del siglo, la inscripción relacional del sentido aparece 
como decisiva. Ella juega, no sin dificultad, como una imposición ontológica entre la visión generada por el desplazamiento, los signos de la alteridad incaptable o ambigua y las conjeturas del sentido. El vector de este juego varía en función de las circunstancias de cada viaje, pero el desplazamiento y la observación orientada persisten como un símbolo de la recreación y la explicación fundamental del mundo. En la cara de la alteridad, los viajantes no pueden presumir el saber y marcar las muchas divisiones que separan a sus propios modelos a ser o de pensar de aquéllos que ellos ladean, que se proponen aprovechar. El constante juego de la identidad y la alteridad sustenta la producción del sentido elaborado por el viaje.

El corazón de las tinieblas puede ser considerado como una de las primeras entradas del discurso moderno de la separación entre las identidades propias de un Occidente capitalista, burgués, imperialista y, por otra parte, británico, y un África salvaje, irreconocible y como un conjunto de signos que el hombre blanco se esfuerza en vano para interpretar y comprender. En la novela de Conrad, el viaje está cargado de un simbolismo cósmico fundamentalmente negativo que Conrad estudiará sistemáticamente en un texto lleno de ambigüedades.

Interpretado por diferentes rejas de la lectura, El corazón de las tinieblas pertenece a la categoría de textos cuyo significado es de gran versatilidad hermenéutica. Prácticamente inagotable, este texto adquiere una relevancia semántica y una densidad interpretativa que varía según el punto de vista crítico adoptado. Sin embargo, lo que parece esencial en El corazón de las tinieblas (visto no sólo como una descripción de un viaje, sino también como una tematización y una problematización de un viaje-aventura) es que la novela, que juega con la dramaturgia del enigma en su centro, pone en alguna hipótesis las premisas morales de la descolonización. Está ahí, puesta en el comienzo de la novela, una advertencia, una explicación que es la importancia de una perspectiva ética y que va a mantener implícitamente la narrativa de Marlow:

La conquista de la tierra, que la mayoría de las veces significa tomarla de aquél que consideremos que tengan un color ligeramente diferente o narices ligeramente más achatadas que los nuestros, no es una cosa hermosa cuando nosotros miramos muy de cerca. Que redime es sólo la idea. Una idea detrás de esto, no un falso sentimental, sino una idea, una creencia abnegada en la idea [...] algo que puede levantarse y doblarse adelante de ella y ofrecerle un sacrificio. (Conrad 1984: 16) 
Conrad introduce una duda epistemológica en la escritura romántica y victoriana de los viajes realizados principalmente en el África central por los escritores británicos. Esta «escritura de viajes» (travel writing) se basa en principios estéticos e ideológicos identificados por Mary Louise Pratt en su libro Imperial Eyes: Travel Writing and Transculturation (Los ojos del imperio: los relatos de viajes y trasculturación). Como base y como punto de partida para su análisis del texto de Richard Burton que lleva el título de Lake Regions of Central Africa (Regiones de los lagos de África central) y publicado en 1860. Pratt identifica tres principios fundamentales: la estetización del paisaje (the landscape is estheticized), la densidad semántica (density of meaning) y la relación de dominio entre quien ve y lo que es visto (the relation of mastery predicated between the seer and the seen) (1999: 343-344). Estos tres principios actúan como medios de comunicación estética de la ideología victoriana de descubrimiento a través de un tropo recurrente que Pratt llama el «rey de todo lo que veo" (the monarch-of-all-I-survey-scene) (1999: 345, 346, 348 y 350). Este tropo persiste en el siglo xx, incluso entre escritores como Alberto Moravia. Por Pratt se puede establecer el funcionamiento ideológico de los tres principios de la siguiente manera: la estética embellece el paisaje y lo hace con carácter retroactivo, una obra de arte va reduciendo exclusivamente al placer estético. El valor cognitivo de la descripción es considerablemente relativizado.

Además, el paisaje se representa como extremadamente rico en términos materiales y semánticos. Pero esta «densidad semántica» es un artífice para engañar porque, en realidad, ella constantemente se refiere a la cultura del viajante. En este caso, la riqueza semántica del paisaje exterior, tal como lo describe Burton, es una referencia explícita y constante a Inglaterra. La relación de dominio entre lo que usted ve y lo que es visto implica que Burton es, al mismo tiempo, espectador, pintor y juez de lo que se ve. Pratt (1999: 345) señala que el tropo del «rey de todo lo que veo» asume «una explícita interacción entre la estética y la ideología, en lo que podría llamarse una retórica de la presencia», que proyecta sobre el espacio la identidad del viajanteescritor. Evidentemente, en esta perspectiva, el problema de la alteridad, que sería un equivalente dialéctico de la identidad del viajante-escritor, no puede ser tan radical para sí mismo, como él plantea para Conrad en El corazón de las tinieblas.

\section{2}

U n escrito de viaje que transgrede los tres principios propios de la retórica de presencia y que se distancia del tropo del «rey de todo lo que veo» destruiría la ideología victoriana del descubrimiento. Conrad es, sin duda, un escritor que rompe con la escritura del viaje victoriano. 
El corazón de las tinieblas tiene el valor de un texto transgresivo que, al menos en el espacio literario inglés, perturba el orden establecido del escrito de viaje. En la novela de Conrad, el viaje es informado por uno de los dos narradores que participan estructuralmente y axiológicamente en la narrativa. El primer narrador inicia al lector en la situación de la narrativa del marco: en Londres, algunos empleados de una compañía naviera (director, abogado y contador) se reúnen para escuchar la narración de Marlow. Lo que Marlow anuncia es precisamente la muy subjetiva descripción de un viaje a través del río Congo, en la que participó como capitán. La descripción se organiza en relación a los avances en el continente negro, que es visto por un barco que tiene circunstanciales turistas, peregrinos, gente que realiza viajes profesionales, así como negros. Este viaje, originalmente concebido como una empresa trivial, se convierte en un descenso a los infiernos para ser narrado con una notable intensidad simbólica y alegórica.

La novela de Conrad, sobre todo, revela la diversidad del espacio que amenaza y subvierte el orden de la escritura del viaje como la caracteriza, basándose en las respectivas críticas de Pratt. Por medio de su texto, Conrad pone en juego la división en espacios y subespacios del mundo diversificado que contiene identidades incompatibles, una impenetrabilitad del otro, una no adhesión de los blancos a los valores de los negros. Estas separaciones espaciales requieren una discusión entre la identidad del mismo y la diferencia del otro. Estas separaciones espaciales son portadoras de posibles conflictos y parecen presidir el discurso de Conrad. Ellas son perceptibles en la dramaturgia discursiva del enigma que presupone una búsqueda de la convicción condenada al fracaso.

En El corazón de las tinieblas, el discurso de viaje se convirtió en una revelación y en el cuestionamiento implícito de la alteridad que está ahí, intensa, violenta e indiferente, pero sobre todo inalcanzable en la forma de cuerpos negros y apariciones humanas. Estas apariciones fantasmagóricas dislocan las certezas de la identidad del sujeto narrante. Conrad pone en escena la superestructura ideológica del capitalismo imperialista y rapaz, frente al juego de fantasmas. En esta alegorización negativa del espacio misterioso, enigmático y adverso, la alteridad se opone como fascinación y horror.

Los términos recurrentes, tales como «corazón de las tinieblas» (heart of the darkness), «tinieblas del corazón» (darkness of heart), «naturaleza salvaje o selva» (wildness), «oscuridad y negritud» (blackness), resumen la perspectiva narrativa y la visión del mundo. Conrad pone en el centro de la búsqueda cognitiva de la narrativa un personaje poco claro, ambiguo y fascinante, uno nombrado Kurtz. Conrad parece querer interrogar el potencial de una 
fraternidad entre negros y blancos, pero la ambivalencia del personaje es tal que escapa a una clara representación de lo que realmente es. Es una alegoría trunca, una encarnación del diablo y de las fuerzas oscuras que, en última instancia, asustan al narrador Marlow.

Para la ironía y la acumulación de rasgos contradictorios en el límite tolerable, Marlow es la persona que Kurtz señala como un retrato de un aventurero y místico, un charlatán y «catador del absoluto», un charlatán mágico venerado por hordas de negros, pero que pacta con lo desconocido. Es importante ver que es precisamente Kurtz que, por su comportamiento y su misión para Sudán, encarna la conciencia y la culpabilidad de los europeos. Él es aquél que toda Europa contribuyó a elaborar: «Toda Europa ha contribuido a la realización de Kurtz, y de a pocos supe que de la forma más adecuada, la Sociedad Internacional para la Supresión de las Costumbres Salvajes le encomendó la elaboración de un informe para su futura orientación» (Conrad 1984: 80).

La presencia invisible de Kurtz, pero elocuente, y su reunión con Marlow, no han resultado en la adquisición de conocimientos por el narrador. Por el contrario, así como el continente negro, como la naturaleza salvaje que Kurtz pertenece a la oscuridad y el horror, y el narrador lo enfrenta como resultado de sus experiencias africanas:

Yo me volveré a la selva, y no al señor Kurtz, que me ha de admitir que era el mismo que está enterrado. Y por un momento me pareció como si yo fuera enterrado en una inmensa tumba de secretos que no pueden ser dichos. Me sentí un peso insoportable oprimindome el pecho, el olor de la tierra mojada, la presencia invisible de la corrupción victoriosa, la impenetrable oscuridad de una noche [...]

Ansiaba por enfrentar solo aquella sombra y hasta ahora no lo sé porque he tenido muchos celos de compartir con cualquiera el negro peculiar de aquella experiencia. (Conrad 1984: 101, 104)

En el fondo de una disforia general, la novela de Conrad señala una alteridad entendida como una diferencia, e incluso como una absoluta sorpresa, por lo que el escritor debe encontrar un sentido. Esto sólo puede ser relacional. Entre la identidad del narrador y la aparición de cuerpos negros en la naturaleza salvaje y el corazón de las tinieblas, la alteridad sería un puente para conectar a los seres humanos en el espacio interrelacional. Es impresionante ver que la aparición de los cuerpos negros confina con una experiencia de los límites de cual el acceso parece interdicto. 
El viaje es una experiencia ante todo ocular. Aunque son evidentes las reacciones negativas, como aquella vez en la que el narrador es dominado por las reacciones de ansiedad y la fascinación. El viaje sólo puede asumir un saber relativo a los hechos potenciales, cuyo ahondamiento podría ser un postulado ético del narrador que coincide con la conciencia moral de Conrad. La cuestión del sentido de la alteridad elaborado por el viaje se convierte entonces en el sentido de un juego relacional de las fuerzas temáticas y semánticas cuya eficacia social y humana podría ser en otro ámbito sociocultural, que la novela de Conrad no se aventura a caracterizar. Conrad se convierte, simbólica y dialécticamente, en un precursor del trabajo de campo etnográfico; lo que demuestra al mismo tiempo que la incursión en los espacios donde evolucionan otras alteridades no sólo es un problema antropológico o etnográfico, sino también político.

3

Bonislaw Malinowski, el etnógrafo quien inventa y practica el trabajo de campo, no sólo
ve en Conrad como su ideal de éxito profesional fuera de las fronteras de su patria y a un ejemplo de complejidad intelectual y psicológica, sino también la realización de un ficticio proceso digno de ser observado y practicado por un etnógrafo en la medida en que no puede tener las herramientas para dominar la complejidad de los otros. El Diario de Malinowski es, entre otras cosas, una presentación sistemática de informes del viaje a las islas Trobriand. Este Diario ocupa un lugar especial en el corpus científico de Malinowski. Él se sitúa en una época difícil en la vida del gran antropólogo, que deja Polonia en 1910 para iniciar estudios de etnología en Londres. Escrito principalmente en polaco con un gran número de frases en otros idiomas, especialmente inglés, alemán, español, griego y latín, además de lenguas vernáculas. El Diario no era destinado para publicación, sin embargo, por iniciativa de su esposa, fue publicado en 1967, traducido del polaco, bajo el título A Diary in the Strict Sense of the term (Un diario en el sentido estricto del término). El tiempo cubierto por el Diario es de septiembre de 1914 a agosto de 1915, y de octubre de 1917 a julio de 1918, correspondientes a las misiones que Malinowski realizó en Australia, Nueva Guinea y en especial en los archipiélagos del sureste de Melanesia (región de Massim, archipiélago de las islas Trobiand).

Si el Diario causó un escándalo, fue porque en él se ve al autor viajante-escritor, al futuro gran etnólogo, al regreso de los problemas psicológicos, instintos sexuales, enfermedades, así como al cuerpo debilitado del escritor. En suma, la experiencia subjetiva de Malinowski, que se describe con precisión, refleja y muestra, como observa Remo Gaidieri (1985), lo que hay «de "impuro" en el viaje etnográfico». 
Sorprende ver que la mayoría de los críticos y comentaristas del Diario de Malinowski establecen paralelismos entre este texto y El corazón de las tinieblas de Conrad. El comentario más elaborado de los dos casos límites fue realizado por James Clifford en su libro The Predicament of Culture: Twentieth-Century Ethnography, Literature, and Art (Desafíos de la cultura: la etnografía, la literatura y el arte del siglo xx) publicado en 1988. Clifford ve en la obra de Conrad la realización de lo que él llama «la subjetividad etnográfica». Él la define como sigue: «La "subjetividad etnográfica" se construye a partir de la observación participante en un mundo de "artefactos culturales" vinculados [...] a una nueva concepción de la lenguaje —o más bien, lenguasmientras los sistemas discretos de los signos» (Clifford 1988: 94-95).

La subjetividad etnográfica sería esta actitud creativa y participante, propia de determinados escritores o etnólogos en los cuales la verdad se relativiza considerablemente; ella se convierte en el resultado de diversas ficciones de diferentes lenguas y diferentes discursos en una perspectiva relacional en que, sin embargo, una lengua y un discurso ordenado que van a dominar, representar y traducir a otras lenguas u otros discursos (Clifford 1988: 112). Al respecto del segundo narrador de El corazón de las tinieblas, Clifford señala que «escrupulosamente yuxtapone diferentes verdades». ${ }^{6}$ Su subjetividad trabaja contra el reconocimiento de una verdad única, recta y ortodoxa. En resumen, la «subjetividad etnográfica» sería una especie de polifonía bakhtiniana englobada por una voz y puesta en la boca de un narrador. Clifford formula esta cuestión en términos ligeramente diferentes:

Marlow, inicialmente, "odia la mentira», pero aprende a mentir, o, comunicarse en el interior de la ficción coletiva y parcial de la vida cultural. Cuenta historias limitadas. El segundo narrador salva, compara estas verdades escenificadas, e (irónicamente) cree en ellas. La voz del narrador «más exterior» de Conrad es una voz de estabilización cuyas palabras no están orientadas para que sospechen de ellas.

(Clifford 1988: 99)

\section{4}

Enos permite reanudar el problema de la alteridad en sus relaciones con los discursos de viajes. $\mathrm{Si}$, como señala Clifford, El corazón de las tinieblas es un "paradigma de la subjetividad etnográfica»

${ }^{6}$ «Truthfully juxtaposes different truths» (Clifford 1988: 99). 
(1988: 100), así como en él se incluye Un diario en el sentido estricto del término, podemos adelantar hipotéticamente que la subjetividad etnográfica corresponde al lado del otro lo que puede ser llamado una alteridad etnográfica. Ella es también una construcción de una relación y presupone aquel juego de ficción y signos que el viajante-observador-escritor emprende necesariamente para estabilizar sus relaciones con el otro. Este último es una construcción, un producto del viajanteescritor. Si el viaje es un operador cognitivo, él cataliza los procesos discursivos de construcción de la alteridad etnográfica, el fondo esencial sin el cual ninguna narrativa de viajes y, sobre todo, ninguno de los discursos de viaje de nuestra modernidad serían posibles.

El acercamiento entre el discurso literario de viaje y el viaje etnográfico nos permite avanzar en la idea de que entre los dos se establece una complementariedad funcional que muestra la polidimensionalidad del problema del otro y de la alteridad. Esta simetría se refiere al hecho de que el escritor de viajes o el viajante-escritor es necesariamente un etnógrafo, así como el etnógrafo no puede estar exento de ser un escritor. Los casos de Conrad y Malinowski son los mejores ejemplos de este estado de cosas. Tanto El corazón de las tinieblas como Un diario en el sentido estricto del término son textos escandalosos, por su carácter híbrido que asumen la doble función de papeles. El problema de los significados de la alteridad se pone de manera más específica, en la medida que ni el otro ni la alteridad puede ser absolutizados. Antes, por el escrito de viaje, puede verse claramente que, como la identidad, que es una estructura polipredicativa, la alteridad se define como un solo y único predicado. La hipótesis de la alteridad etnográfica muestra que ella es ante todo una ficcionalización del otro, una relativización de su absoluto subjetivo como diferencia. Yo diría que esta alterización es una «etnografización», o sea, un realce de su diferencia, no sólo subjetiva, sino también social y antropológica, en el sentido de que está vinculada a la identidad colectiva del grupo social al que pertenece y por la cual se forja.

5

【os modos de funcionamiento de la alteridad etnográfica son similares a algunas de las problematizaciones situadas en la alteridad en las construcciones discursivas que, en primer lugar, reducen el otro a un otro, expresando la «alteridad radical». Estamos aquí en el área de "figuras de la alteridad». Baudrillard y Guillaume abordan la compleja cuestión de la alteridad en una perspectiva innovadora, marcando especialmente el sentido y la función de la «alteridad radical». Su teoría servirá como una herramienta crítica para ilustrar las diferentes formas de la alteridad 
en los discursos de viaje. Lo que es claramente colocado en Les figures de l'altérité (Las figuras de la alteridad) —de Baudrillard y Guillaume — es la distinción entre el otro como un otro y la alteridad radical. Guillaume sitúa este problema como sigue: «En todo otro hay un otro - lo que no es yo, lo que es diferente de mí, pero puedo entender, y asimilar — y hay también una alteridad radical, imposible de asimilar, incomprensible e incluso impensable» (Baudrillard 1994: 10).

Las sociedades occidentales homogeneizaron lo que era radicalmente heterogéneo, lo que es una verdadera rareza, a saber, la alteridad. Guillaume estima que a través de «ficción mixta [...] es algo que se construye a partir de un real y que el siguiente es dosificado con un cierto grado de imaginación, de ficción» (Baudrillard 1994: 49). Podemos confirmar esta posición del problema refiriéndonos a ciertos discursos de viaje. Pongamos en primer lugar los discursos que tratan de asimilar y entender al otro en el punto en que se convierte en una entidad existencial y social domesticada, domada y conocible. En algunos discursos de viaje con frecuencia ocurre que el otro y la alteridad están representados a partir de la experiencia del ocular transcribida por la narrativa de manera superficial, a menudo mediante el uso de estereotipos y generalizaciones lleno de sintetizadores.

Así como Almeida Garrett ve a Laura, la mujer que es este otro, en la que el viajantenarrador establece una estricta descripción ocular:

\footnotetext{
Laura no era alta o baja; era fuerte sin ser gorda, y delicada sin delgadez. Los ojos de un color avellana, puros, aterciopelados, grandes, vivos, llenos de majestad cuando irabanse; de tal dulzura cuando se ablandaban, que es difícil decir cuándo eran más bellos. El cabello, casi del mismo color, tenía lo más, un reflejo de oro, tambaleante, que resplandecía en el sol, o más bien, brillaba; pero el espacio no era siempre, ni en todas las posiciones de la cabeza: cabeza pequeña, el modelo de más clásico de las antiguas estatuas, como en un regazo de inmensa nobleza, que vivía en armonía con la perfección de las líneas de los hombros.

La cintura, corta y estrecha, pero sin exageración, se veía que era así por naturaleza sin la menor contrafeición de arte. El pie no era exiguo como nuestra fabulosa Península, tenía proporción como la Venus de Medici. (Garrett 1954: 295-296)
}

La descripción de los árabes por Chateaubriand, Itinéraire de Paris à Jerusalém (Itinerario de París a Jerusalén), que ha sorprendido por las opiniones y sentencias extrapolantes: 
Los árabes, en todas las partes donde vi en Judea, Egipto e incluso en Barbaria, me parecían antes de alta, más que de baja estatura. Su paso es alto. Ellos son bien hechos y delgados. Tienen cabeza ovalada, la frente alta arquelada, la nariz aguileña, grandes ojos en forma de almendras, los ojos húmedos y singularmente dulces. Nada anunciaría en ellos el salvaje, si llevase siempre la boca cerrada, pero que llega a hablar, se oye una lengua ruidosa y de firme aspiración, y se ven largos dientes de blancura deslumbrante, como de chacales y onzas: diferentes de los salvajes de América, cuya ferocidad se encuentra en el ojo, y la expresión humana, en la boca. (Chateaubriand: 301-302)

En Un barbare en Asie (Un bárbaro en Asia), Henri Michaux, de la misma manera, no escapa a los estereotipos o generalizaciones injustas: «El árabe, violento en su lengua eructada, el árabe duro y fanático, el turco conquistador y cruel son también personas con nauseabundos perfumes, jalea de rosas y loukoums ${ }^{7}$ (Michaux 1967: 39).

Estos ejemplos indican la intención simplista y generalizante de los viajantes-narradores. En sus discursos, el otro aparece no como individuo sino como un actor colectivo y, por lo tanto, naturalmente reducible a otras personas. El personaje de Laura, descrito por Almeida Garrettt, pertenece a la categoría de la mujer excepcionalmente hermosa, tan hermosa como la Venus de Medici. Los árabes de Chateaubriand son salvajes, a pesar de su noble apariencia. Es su lenguaje «ruidoso y muy aspirado» que los hace salvaje. Para H. Michaux, los árabes, que son violentos por su «lenguaje eructado», y los turcos, que son conquistadores y crueles, son repugnantes por sus «nauseabundos perfumes» e infantiles a causa de que consumen «jalea de rosas y loukoum».

\section{La ficción mixta y la construcción de la alteridad radical 1}

Ta formación de la alteridad radical es a través de la divulgación y del realzamiento de _ciertos rasgos significativos o ciertos comportamientos, individuales o colectivos, que, intencionadamente y por el deseo de captar la verdadera diferencia, escapan de los estereotipos.

\footnotetext{
7 Confite oriental hecho de una masa aromatizada llena de azúcar refinada (N. T. de la versión en portugués).
} 
Son irreductibles a ese comercio fácil de escritos de viaje, como hemos visto desde el otro y otro. Comercio que incluye ideológicamente todas las diferencias disminuyéndolas en los estereotipos y, por tanto, asimilando a el otro y un otro. En algunos discursos en los que el viaje es muy problematizado, vemos el intento de complejizar la cuestión de la identidad y de la alteridad. Son las ficciones mixtas que demuestran mejor la complejidad de la alteridad radical. A diferencia del proceso de homogeneización, las ficciones mixtas mezclan el real y el imaginario en un gesto etnográfico destinado a hacer emerger la alteridad radical.

La expresión de la alteridad radical, que es también una alteridad etnográfica en el sentido que la definimos, se puede anclar en el testimonio etnográfico. Esto se pude apreciarse en África fantasma, de Michel Leiris. El sacrificio de los pollos es completamente descrito el 4 de octubre de 1932.

La distribución de los pollos: después de cada tres saludos, que recibe el volátil de Malkam Ayyahou, diciendo «djeba» (ofrenda), le da y, a continuación, ella baila con el bate en la mano y el pollo en la cabeza. La danza colectiva. Salida para un punto diferente del jardín, elegido como el lugar de sacrificio. Los adeptos se quejan de $r a k i i^{8}$; Griaule manda buscarlo.

Trance de Malkam Ayyahou, que se oculta detrás de Chamma. Es Wassan Galla. En su foukkara, esto zar declarado, entre otras cosas, "Comedor de Enqo-Bahri» Enqo Bahri saluda Malkam Ayyahou, después de su trance. Luego, llevando el carnero entre sus piernas, como si estuviera a caballo encima de él, lo presenta a Malkam Ayyahou. Este, sentado, toma el carnero por los cuernos y lo expone a una fumigación de incienso. Ella acostada a seguir por la tierra pone su pie derecho sobre su cabeza, que deseando una derrocada semejante a los enemigos de la Enqo Bahri. (Leiris 1981: 409)

La naturaleza mixta de esta descripción reside en el hecho de que la escritura de Leiris es, en primer lugar y ante todo, un periódico. El escritor-etnógrafo que también le agrega el ritual, los gestos, la proxémica y la narrativa, introduciendo palabras de la lengua vernácula.

El ojo etnográfico de Claude Lévi-Strauss, en Tristes trópicos, se vuelve también hacia esto, y quizás sobre todo por las multitudes que viven en las grandes ciudades de la India. La

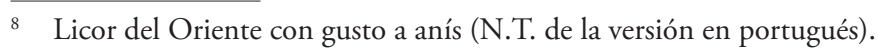


alteridad radical de los indios (naturales de la India) será aprendida en la escala de las enormes multitudes que participan de manera sufrida de la vida de las «zonas», porque, como LéviStrauss menciona: «Las grandes ciudades de la India son una zona», y continúa:

Pero lo que nos da vergüenza como un fetiche, que consideramos como una lepra, aquí es el hecho de que la ciudad sea reducida a su más simple expresión: la aglomeración de personas cuya única razón de ser es la de acumular millones, cualesquieras que sean las condiciones reales. La suciedad, desorden, promiscuidad, aglomeramiento, ruinas, casillas, barro, suciedad, estado de ánimo, heces, orina, pus, secreciones, purulencias: todo lo que de la vida da contra la vida urbana, en la defensa parece estar mejor organizada, todo lo que odiamos, en contra de la que nos protegemos a un precio tan alto, todos estos derivados de la cohabitación, aquí no son nunca un límite. Por el contrario, forman el entorno natural que la ciudad necesita para prosperar. Para cada individuo, la calle, callejón o acceso directo es de lo único que dispone, en los que descansa, duerme y donde saca sus alimentos de la basura pegajosa. Lejos de rechazar eso, ésta adquiere una especie de estado de familia por el simple hecho de haber sido expulsada, defecada, pisada y tratada por tantos hombres. (LéviStrauss 1955: 113)

En la extensión de la tradición filosófica de viaje, Lévi-Strauss define un marco y construye un discurso, cuya poesía se caracteriza por la fusión de elementos dispares. En su ficción mixta, el narrador-viajante combina factores sociológicos (el estudio del hecho urbano), etnológicos (comportamiento colectivo de las personas «naturalmente» miserables), y comparativos (la oposición entre «nosotros», el Occidente próspero y sabio, y el Oriente de los otros, los indios, pobres y aglomerados por el sacar la comida directamente de la «basura pegajosa»). Esta identidad colectiva de la condición pseudoexistencial de estas personas de la India y la miseria que tiene su sede en los residuos de todo tipo, también revela la alteridad radical. Esta diferencia es intrasladable para un occidental.

Cabe destacar que la enumeración adquiere en este discurso un estatuto particular, la verdadera diferencia. Todos los objetos y gestos repugnantes parecen pertenecen únicamente a los lugares privados en la India. Sin duda, podría situarse en Nápoles, Río de Janeiro, en barrios 
o favelas en América Latina. Sin embargo, la enumeración reúne elementos irrepetibles, propios de los lugares en la India, como las heces, la promiscuidad, el aglomeramiento y las purulencias.

F $\mathrm{n}$ algunos discursos, la manipulación textual y discursiva da al viaje una función de - 0 operador cognitiva, problematizándola al espacio. Desde Kant, sabemos que el espacio no es un concepto empírico. Se define por el discurso del observador desde el lugar de observación. Viajando se conoce por sus propios ojos un espacio supuestamente objetivo. En realidad, esta fórmula podría tomar otra forma en los textos con una fuerte dosis de discontinuidades de los recortes, de la fragmentación y de los montajes.

Viajar es conocer el espacio a través de los ojos del texto. Ésta parece ser la ficción mixta en Le città invisibili (Las ciudades invisibles), de Italo Calvino; Mobile, de Michel Butor, y en África alfabética, por Walter Abish. Estos libros son la síntesis de uno o varios viajes. A ejemplo de la metáfora "tramada» (filée) de los surrealistas, estos viajantesescritores ofrecen viajes textualmente tramados. Textualmente discontinuos, fragmentados y totalmente montados. Sus discursos de viajes son entonces metadiscursivos en la medida en que la escritura estaba allí para mirar a sí misma, y el viaje tiene un estatuto particular espacio-temporal. El viaje trabaja allí como texto, siempre abierto y potencialmente infinito, un nuevo paso en esa posición de los discursos de los tres narradores-manipuladores. No hay registro de las impresiones del viaje. Hay, sí, viajes textuales que se refieren a recuerdos comprendidos en la categoría mental e intelectual, similares a la memoria, al deseo y la mirada. El viaje es esta substancia rica, siempre in statu nascendi, determinada-indeterminada, este iceberg, cuya parte sugirió Calvino, Butor y Abish, que se empeñaron en mostrar.

En esta configuración de las ficciones mixtas, las relaciones con la alteridad se estructuran de manera particular: por una negociación constante del sentido, por una colocación intencional de los signos relacionales, cuya presencia genera los sentidos de la alteridad etnográfica, mientras proceden la composición dinámica de sus ficciones mixtas. Es a este precio, por lo tanto, que ellos producen la radicalización de las alteridades en el ir y venir de la mimesis, de la mathesis y de la semiosis. 
$7 \mathrm{n}$ Las ciudades invisibles, de Calvino, el gran viajante Marco Polo cuenta sus viajes Eal emperador de los tártaros. Las narrativas de Marco Polo, a través del emisario del emperador, se refieren a una serie de ciudades. El narrador deslumbra al emperador, aunque él no cree en todo lo que Marco Polo cuenta. El vértigo de estas narraciones discontinuas produce un efecto de hechizo en los lectores. Todas las ciudades narradas tienen nombres femeninos, todas ellas son extrañas, desconocidas e invisibles en los mapas. Ellas evolucionan en el discurso y son de difícil interpretación. Probablemente existen en la memoria, en el sueño y el deseo. Refiriéndose constantemente no sólo a la memoria y la imaginación y a las imágenes memoriales, sino también para el discurso de Marco Polo. El desfile de las ciudades invisibles desemboca en la metrópoli moderna e infernal, donde Calvino sugiere buscar islotes, que no sean del infierno. La aparición ficticia, así como el establecimiento de los rasgos de las ciudades invisibles las escribe en un campo de una forma simbólica y global de la humanidad.

La mezcla de ficciones de Calvino se obtienen adjuntando elementos descriptivos discursivos, casi etnográficos, que significan la singularidad de cada ciudad. Las ciudades, precisamente a causa de su extrańeza señalan para el pueblo, las alteridades. Son alteridades utópicas, tal como se definen en su relación con el sueño, con el deseo, con los proyectos de vida y en relación al espacio de fuertes connotaciones simbólicas y alegóricas. Con respecto al propio narrador y al destinatario de las narrativas, estas ciudades abrigan a los otros, las alteridades anónimas, masas mudas en las que no es permitido hablar. Su silencio no descarta que sean signos para expresar su apego al espacio y la vida. El sentido de las alteridades en cuestión sería algo así como la figura de la utopía perdida para siempre, viva en el deseo más allá del infierno urbano moderno: «Todas las cosas que veo ganan sentido en un espacio de la mente que reina en la misma calma que existe aquí, el mismo gris, el mismo silencio recorrido por el susurro de las hojas» (Calvino 2003: 99).

Calvino produce, en Las ciudades invisibles, una especie de cosmogonía urbana total. Los seres humanos que la habitan se comportan de manera tan extraña como lo insólito de lo que cada ciudad está hecha. Sus alteridades son implícita e irónicamente fijas. "Éstas son las ciudades invisibles cuya visibilidad muestro», parece querer decir Calvino. Y sus habitantes, similares a nosotros, viven en una utopía que les podríamos envidiar. 
Estamos en el infierno de la ciudad moderna y de la aldea global porque perdemos nuestra utopía. Podríamos haber sido como ellos, felices o infelices, pero en un espacio diferente de las ciudades invisibles, que figuran en sentido ambiguo y tan rico como la condición humana.

Cuál es la radicalización de la alteridad de América? El viaje de Michel Butor a través de cincuenta estados nos habla de qué sistemáticamente, pero indirectamente. Mobile es el enorme montaje de un enorme espacio que no deja de cambiar. En el espacio de unas pocas páginas del libro, pasa de un estado a otro, donde es bienvenido. Debido a que la palabra bienvenue (bienvenido) es el principal embreante del texto. "Bienvenue au Texas», «Bienvenue au Tennessee», «Bienvenue en Oklahoma» y así sucesivamente.

Butor crea un fascinante patchwork en que puede ser vista una imagen de los Estados Unidos rica en hechos, gestos, costumbres y en las palabras con la mezcla de signos onomásticos, ya sea con nombres históricos o apelativos comunes, citas de documentos históricos, crónicas, fragmentos de recortes de artículos (en los periódicos de América), fragmentos de textos literarios o fracciones de los diálogos. Se trata de un viaje casi fantástico, textualmente homologado por los efectos del montaje, de la fragmentación del avance en el tiempo y del espacio. La ficción de Butor es mixta por los elementos que indicamos. Ella se afirma como el discurso de un viaje que cruza todo un continente con naturalidad y, con él, una serie de contingencias y requisitos verbales, gestuales y visuales. La estructura del texto representa la casualidad de los encuentros y lo que el narrador principal del montaje escucha y registra, lo que ve y entiende, lo que introduce al texto después de una selección semiótica de la masa de los documentos compilados.

En este montaje de espacios seccionados en cincuenta estados, los estadounidenses aparecen como un pueblo feliz, dueño de un vasto territorio con una historia corta, pero gloriosa, que condujo directamente a la democracia. Los ciudadanos son a menudo personas de otros países. Participan del melting pot, cuyo sentimiento es también proveniente del texto. Pero los estadounidenses son también los indios, autóctonos, que comprenden un mosaico de distintas razas. El multilingüismo del país está fuertemente resaltado por Butor. A menudo, él elige los títulos de periódicos en diferentes lenguas para representar la estratificación poliétnica de los EE. UU. El viaje de Butor es no sólo moderno y modernista, 
sino también etnográfico e histórico. Por eso, esta versatilidad requiere una alteridad radical. Ella es correlativa a la identidad de América, cuyos elementos tienen su punto de partida en el «sueño americano». Y más allá de la convergencia, ese sueño se realza en el texto por la exaltación y la expresión de inmenso espacio, pero al mismo tiempo Butor introduce elementos negativos en su montaje: el racismo, la violencia y los vergonzosos episodios en la historia, como la caza de brujas. Podría decirse que la alteridad estadounidense es ideológicamente homogénea y pragmáticamente funcional. Pero vista de más cerca, la alteridad radical que se puede ver desde Mobile es desequilibrada. Ella es la heredera de peso de la historia llena de acontecimientos con sus consecuencias. Por lo tanto, es escindida, llena de susceptibilidad, pero quizá ésta deriva de un hecho subrayado por uno de los hechos conductores de Mobile:

Lo que tenía de amedrentador este continente no eran sólo sus lianas envenenadas...

Sus robles envenenados, zumaques venenosos, ponzoñosas serpientes, flechas de indios envenenadas...

Lo que tenía de amedrentador, antes de cualquier experiencia, era la existencia misma de este continente, salida de más allá del horizonte, exactamente donde no debería haber sido. (Butor 1962: 105-107)

5

T a «novela» África alfabética, de Walter Abish, no es contable. A pesar de que hay eventos _y hechos narrados y narrativos es un proyecto que expone metadiscursivamente el conjunto de África en lengua inglesa en su totalidad, simulando todo el alfabeto. El autor explica todo lo que es africano a la cronología de las letras, de la A a la Z, y de la Z a la A. Por ello, forma conjuntos de palabras, de sintagmas, de términos relevantes para el conocimiento de África, no sólo en inglés, sino también en varias lenguas africanas. En resumen, están sujetos a la saturación de África por el lenguaje ordenado alfabéticamente. Lo que es fascinante en África alfabética es el vértigo de la repetición que se traduce en monotonía, la «monotonía» de África, tema agotado hasta el extremo. Por lo tanto, el metanarrador desencadena factores desde la letra $S$, que prevé, entre otras cosas, la siguiente secuencia: 
Los mismos esclavos, mismo sueño, misma enfermedad del sueńo, misma masacre, misma fumarada, misma superficie lisa, misma obscenidad, mismas serpientes, mismas fotos, mismo escarnio, mismo escenario social, mismos estudios, mismo sistema solar, mismos soldados, mismo sos, misma investigación, mismo espacio, mismas chispas, mismas lanzas, mismo espíritu, mismo esfínter, mismas espirales, mismas arañas, mismos espectáculos teatrales, mismos desfiles, mismas declaraciones [...] misma mierda, misma represión, misma supremacía, mismos excedentes, mismos ambientes, mismo recargo, misma supervivencia, mismos sospechosos, mismo suspenso, mismas golondrinas suajilis, mismos cisnes, mismos cambios, mismos exámenes, mismos suazis bronceados [...] mismos juramentos, mismos símbolos a la moda, misma esgrima, mismas sílabas, mismos silogismos, misma simbiosis, misma simetría [...] mismas sorpresas. ${ }^{9}$ (Abish 1974: 101, la traducción es nuestra)

Al hacer la lectura de Abish, uno se queda impresionado por la riqueza del catálogo de las cosas de África. La ruta del alfabeto en ambas direcciones se encierra durante la siguiente observación: «otra África, otro alfabeto» (ídem: 152). Esta manipulación del texto produce un efecto de monotonía y de repetitividades existenciales. Podría tenerse la impresión de que se trata de recuerdos ordenados de un turista desencantado que no volverá a África después de catalogadas sus impresiones de viaje.

La cuestión de la alteridad radical reside en el nivel de percepción del pueblo de África por un escritor y viajante cosmopolita. Y esa percepción es muy lúdica, ella cae en broma verbal a la que ofrece Abish. Manipulando sus conocimientos de África, éste construye un mobile que va en todas direcciones, pero sistemático y concéntricamente, al igual que los pétalos de una rosa. África alfabética es una simulación del descubrimiento del continente africano. Ella tiene éxito y, como tal, representa la forma de los átomos que constituyen el gran edificio de África. La alteridad de África forma parte de esa red textual. Esto significa la diferencia como un conjunto ordenado de las partes con el fin de construir la ficción de una guía turística o de una enciclopedia de África.

9 "[...] same slaves same sleep same sleeping sickness same slaughter same smoke same smooth surface same smut same snapshots same sneers same social scenes same studies same solar system same soldiers same SOS same searching same space same sparks same spears same spirit same spnincter same spiral same spiders same staged shows same standstills same statements [...] same shit same suppressions same supremacy same surplus same surroundings same surtax same survivors same suspects same suspense same Swahili swallows same swans same swaps same swarms same swarthy Suazis [...] same sworn statements same swinging symbols same swordsplay same sylables same syllogismos same symbiosis same symmetry [...] same surprises $[\ldots] »$. 


\section{Conclusión}

Fl problema que abordamos se define como representaciones discursivas de viajes y de una manipulación de los distintos saberes que vienen, más concretamente, en la construcción de la subjetividad y alteridades etnográficas. Éstas se basan en procesos discursivos variables, pero sobre todo mixtas. El sentido de la alteridad se deducirá de las construcciones narrativas y discursivas complejas y polivalentes que expresan el otro y los otros en las diferentes modalidades de su participación en una comunidad humana, y frente a los otros. La alteridad es así pensada como correlación de la identidad en una relación interhumana.

En los textos en discusión, desde Conrad hasta Abish, se distingue como la posición de los narradores, así como sus operaciones discursivas y textuales, determinan el sentido de la alteridad puesta en un juego relacional. La semiosis es a su vez determinada por la dinámica de la inagotable triple relación: narrador-viajante, espacio, y alteridad. El viaje en la alteridad moderna presenta pares múltiples y funcionales en la medida en que es, en primer lugar y ante todo, un operador cognitivo y generador de conocimientos diversos y de metadiscursos. 


\section{BIBLIOGRAFÍA}

AвISH, Walter

1974

Alphabetical Africa. Nueva York: A New Directions Book.

BARTHES, Roland

1978

Leçon. París: Seuil.

BAJTin, Mijail

1997

Estética de la creación verbal. Traducción de Maria Galvão Ermantina G.

Pereira. 2. ${ }^{\mathrm{a}}$ ed. São Paulo: Martins Fontes.

Baudrillard, Jean y Marc Guillaume

1994

Les figures de l'Altérité. París: Descartes \& Cia.

Butor, Michel

1962

Mobile: étude pour une représentation des États-Unis. París: Gallimard.

Calvino, Ítalo

2003

Las ciudades invisibles. Río de Janeiro: Editora Globo.

Canetri, Elias

1987

Las voces de Marrakech. Traducción de Marijane Lisboa. Porto Alegre:

L\&PM.

Clifford, James

1988

The Predicament of Culture: Twentieth-Century EthnogRaphy, Literature and Art. Cambridge, Massachusetts/Londres: Harvard University Press.

Conrad, Joseph

1984 [1902] El corazón de las tinieblas. Traducción de Marcos Santarrita. 2. a ed. São Paulo: Brasiliense (tít. original: Heart of Darkness. Londres: Penguin Books). 
DARCy Ribeiro, Maira

1980 Río de Janeiro: Brasil Civilización, 1976. Traducción del portugués por A. Raillard. París: Gallimard.

Garrett, Almeida

1954 Viagens na minha terra. Lisboa: Librería Sá da Costa.

GinsBerg, Allen

1977 Journaux indiens. Traducción de P. Mikriammos. París: Christian Bourgeois, UGE.

GUIDIERI, Remo

1985 Journal d'ethnographe. París: Seuil.

LeIrIs, Michel

1981 L’Afrique fantôme. París: Gallimard.

LÉvi-STrauss, Claude

1955 Tristes tropiques. París: Plon.

1957 Tristes trópicos. Traducción de Wilson Martins. Sáo Paulo: Anhembi.

LE ClÉZIo, J.M.G.

1975 Voyages de l'autre cote. París: Gallimard.

Michaux, Henri

1967 Un barbare en Asie. París: Gallimard.

PratT, Mary Louise

1992 Imperial Eyes: Travel Writing and Transculturation. Londres/Nueva York. Routledge.

1999 Os olhos do Império: relatos de viagem e transculturação. Traducción de Hernani Jézio Gutierre Bonfim. Bauru, SP: Edusei. 
Seixo, Maria Alzira (org.)

1997 A viagen na literatura. Lisboa: Europa-América Publicaciones, pp.235263.

SERres, Michel

1986 «Un Voyage au bout de la nuit». Critique, N. ${ }^{\circ}$ 263, abril. París. 